UCRL-JC-120184

CONF-941144- -173

\title{
Modeling of Microencapsulated Polymer Shell Solidification
}

\author{
T. Boone \\ L. Cheung \\ D. Nelson \\ D. Soane \\ G. Wilemski \\ R. Cook
}

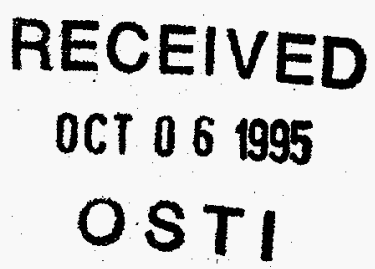

This paper was prepared for submittal to the Symposium of Hollow and Solid Spheres and Microspheres

Materials Research Society Meeting

Boston, MA

November 28-December 2, 1994

March 8, 1995

This is a preprint of a paper intended for publication in a journal or proceedings. Since changes may be made before publication, this preprint is made available with the understanding that it will not be cited or reproduced without the permission of the author. 


\section{DISCLAIMER}

This document was prepared as an account of work sponsored by an agency of the United States Government. Neither the United States Government nor the

* University of California nor any of their employees, makes any warranty, express or implied, or assumes any legal liability or responsibility for the accuracy, completeness, or usefulness of any information, apparatus, product, or process disclosed, or represents that its use would not infringe privately owned rights. Reference herein to any specific commercial product, process, or service by trade

name, trademark, manufacturer, or other wise, does not necessarily constitute or imply its endorsement, recommendation, or favoring by the United States Government or the University of California. The views and opinions of authors expressed herein do not necessarily state or reflect those of the United States Government or the University of California, and shall not be used for advertising or product endorsement purposes. 


\section{DISCLAIMER}

Portions of this document may be illegible in electronic image products. Images are produced from the best available original document. 
TRAVIS BOONE*, LISA CHEUNG*, DONALD NELSON*, DAVID SOANE*, GERALD WILEMSKI**, AND ROBERT COOK ${ }^{* *}$

"Soane Technologies, Inc., Hayward, CA 94545

Lawrence Livermore National Laboratory, P.O. Box 808, Livermore, CA 94551

\section{ABSTRACT}

A finite element transport model has been developed and implemented to complement experimental efforts to improve the quality of ICF target shells produced via controlled-mass microencapsulation. The model provides an efficient means to explore the effect of processing variables on the dynamics of shell dimensions, concentricity, and phase behavior. Comparisons with experiments showed that the model successfully predicts the evolution of wall thinning and core/wall density differences. The model was used to efficiently explore and identify initial wall compositions and processing temperatures which resulted in concentricity improvements from 65 to $99 \%$. The evolution of trace amounts of water entering into the shell wall was also tracked in the simulations. Comparisons with phase envelope estimations from modified UNIFAP calculations suggest that the water content trajectory approaches the two-phase region where vacuole formation via microphase separation may occur.

\section{INTRODUCTION}

Highly spherical polymer shells with uniform wall thicknesses are used as Inertial Confinement Fusion (ICF) targets. Current targets for the U.S. ICF Program are built around polystyrene microshells no more than $0.5 \mathrm{~mm}$ in diameter. As more powerful lasers come on-line, microshells with diameters up to $2 \mathrm{~mm}$ will be needed. ${ }^{1}$ One technique for producing such shells is controlled-mass microencapsulation which consists of forming a water-oil-water emulsion shell using a triple orifice droplet generator. The solvent from the oil phase, in which polymer is dissolved, diffuses into the adjacent external and core water phases, resulting in a hardened polymer shell, as shown in Figure 1. In addition to the diameter and wall thickness specifications, shells must have excellent concentricity and sphericity. Shells must also be free of vacuoles, which are micron-scale voids believed to result from the microphase separation of trace amounts of water within the oil-rich wall during shell hardening.

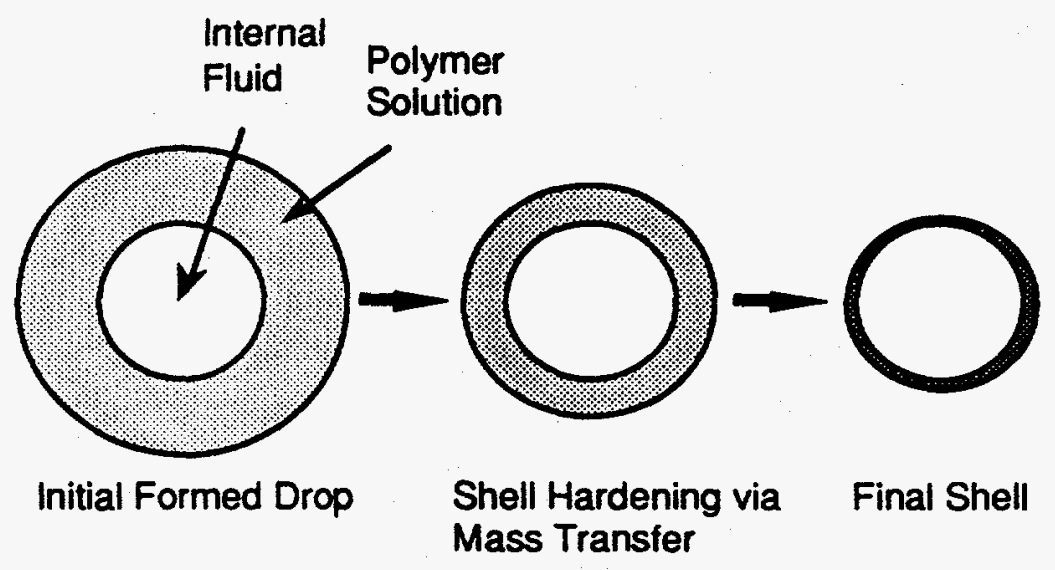

Figure 1. Schematic of microencapsulated shell solidification process

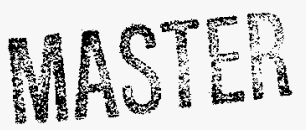


There are many variables that affect the shell solidification dynamics and vacuole content. These include polymer and solvent type, initial compositions of each phase, foreign particulate levels, orifice flow rates, temperature, and agitation. The objective of the modeling effort is to aid in achieving desired target quality by providing an efficient means to explore the effect of processing variables on the dynamics of shell dimensions, concentricity, and phase behavior.

\section{THE MODEL}

Our model simulates the transient evolution of radial composition profiles and wall thickness of a spherically symmetric shell. Mass transfer within the wall is assumed to be diffusive; no fluid circulation within the wall is considered. In a fluid of $N$ independent chemical components, there are $N-1$ independent diffusion fluxes $J_{i}$ defined by

$$
J_{i}=\rho_{i}\left(\bar{u}_{i}-u\right)
$$

where $\rho_{i}$ is the mass of component $i$ per unit volume and $\bar{u}_{i}$ is the local mean velocity of $i$. Equation (1) holds in a reference frame in which the fluxes are measured relative to the mass average velocity $u$ where

$$
u=\sum_{i=1}^{N}\left(\rho_{i} \overline{u_{i}}\right) / \sum_{i=1}^{N} \rho_{i}
$$

The time and space dependence of the mass concentrations is described by equations of the form

$$
\frac{\partial \rho_{i}}{\partial t}=-\nabla \cdot\left(J_{i}+\rho_{i} u\right) .
$$

From the theory of irreversible thermodynamics, ${ }^{2} J_{i}$ depends on the $N-1$ independent concentration gradients and can be expressed for an isothermal system as

$$
J_{i}=-\sum_{j=1}^{N-1} D_{i j} \nabla \rho_{j} .
$$

The multicomponent diffusion coefficients $D_{i j}$ are related to Onsager phenomenological coefficients $L_{i j}$ by the equation ${ }^{2}$

$$
D_{i j}=\frac{1}{T} \sum_{k=1}^{N} L_{i k}\left(\frac{\partial \mu_{k}}{\partial \rho_{j}}\right)
$$

where $\mu_{k}$ is the chemical potential of species $k$ and $T$ is the absolute temperature.

The principal practical difficulty in using this formulation to treat diffusion phenomena arises from the general lack of knowledge concerning the magnitude and concentration dependence of the $L_{i j}$. Although the $L_{i j}$ can, in principle, be evaluated from their time-correlation-function expressions ${ }^{2}$ by means of molecular dynamics simulations, this would entail considerable effort to cover the wide concentration ranges spanned during shell formation. A more promising approach may be to evaluate the $L_{i j}$ by emulating $A k c a s u^{3}$ who has used the random phase approximation to express the interdiffusion coefficient of pseudo-binary polymer mixtures and solutions in terms of more fundamentally accessible component mobilities and partial structure factors. Computer simulations could then be used to selectively test these approximate analytical results. The 
thermodynamic factors $\partial \mu_{i} / \partial \rho_{j}$ can be computed from an accurate expression for the free energy of mixing of the multicomponent system, which we obtain using the UNIFAP methodology. ${ }^{4}$ The UNIFAP temperature and free-volume dependencies were modified to improve the water/solvent solubility predictions. Until the comprehensive multicomponent treatment is available, we have implemented a more empirical approach to help interpret and understand the experimental results.

In our current simplified approach we use mass fractions as the concentration variables, neglect all of the diffusional cross terms, and approximate the remaining diagonal terms using the FujitaDoolittle relation,

$$
\log \frac{D i}{D_{0}}=\frac{v_{s}}{\left(A+B v_{s}\right)}
$$

Here, $D_{i}$ is the pseudo-binary diffusivity of solvent $i$ in the polymer solution, $D_{0}$ is the infinite dilution diffusivity of $i$ in the pure polymer, $v_{S}$ is the total solvent volume fraction, and $A$ and $B$ are semi-empirical parameters. This procedure neglects all effects of non-ideal and immiscible solution behavior on diffusion that would enter through the thermodynamic factors in equation (5). This will be most significant of course, for the transport of water into and out of the drying shell.

Mass transfer fluxes between phases (core/wall/external) are of the form

$$
J_{i}=k_{m} \rho_{i}\left(w_{i}^{i n t}-w_{i}^{\text {dest }}\right)
$$

where $k_{m}$ is the mass transfer coefficient, $w_{i}{ }^{i n t}$ is the weight fraction of $i$ in a saturated thin film at the interface, and $w_{i}$ dest is the bulk weight fraction of $i$ on the destination side of the interface. The mass transfer coefficient for each component is estimated using the Chilton-Colburn ${ }^{5}$ relation and verified experimentally. The value of $w_{i}^{\text {int }}$ is computed from the equation,

$$
w_{i}^{i n t}=v_{i}^{\text {orig }}\left(\frac{\sum_{j \neq i}\left(\frac{w_{j}^{\text {dest }}}{1-w_{i j}^{s a t}}\right) w_{i j}^{s a t}}{\sum_{j \neq i}\left(\frac{w_{j}^{\text {dest }}}{1-w_{i j}^{s a t}}\right)}\right)
$$

where $v_{i}$ is the volume fraction of component $i, w_{i j}$ sat is the saturation weight fraction of $i$ in another component $j$, and the superscripts orig and dest refer to the origin and destination sides of the interface, respectively. This definition assumes that the amount of a component $i$ present at the interface is based on the availability of $i$ on the origin side of the interface and on the extent to which $i$ can saturate a thin film of all other components $j$ on the destination side of the interface. Saturation values are taken from literature on pair miscibilities.

The spherically-symmetric mass transfer equations describing the system are af the form

$$
\rho\left(\dot{w}_{i}+u_{r} \frac{\partial w_{i}}{\partial r}\right)=\frac{1}{r^{2}} \frac{\partial}{\partial r}\left(r^{2} \rho D_{i} \frac{\partial w_{i}}{\partial r}\right)
$$

where $\dot{w}_{i}$ is the time derivative of the weight fraction of $i$ and $u_{r}$ is the bulk mass-average velocity at radial position $r$. The temporal evolution of the system is simulated using an iterative 
Galerkin $^{6}$ finite element scheme on a non-uniform 21 -node grid; the nodes are more densely spaced toward the outer shell wall. The external flux at the outer wall boundary is calculated via equation (7); a no-flux boundary is maintained at the inner wall/core (quiescent) boundary. For each time step, the external mass transfer of each component is calculated and the outer wall position is adjusted (shrinks) to conserve pure component densities within the wall, under the assumption of ideal mixing. The boundary weight fractions of each component are then adjusted independently based on Jacobians until the remaining wall mass is conserved and self-consistent overall profiles of bulk velocity, density, and diffusivity are converged upon. With each iteration, weight fraction profiles are calculated by solving the tridiagonal finite element matrices using standard matrix calculation techniques. A typical simulation run of $1000 \mathrm{~s}$, using a time step of $0.1 \mathrm{~s}$, requires approximately one hour on a 486 personal computer. The primary outputs of the model are the evolution of shell diameter and wall composition profiles. From these quantities, the wall thickness, density, and phase behavior are also calculated.

\section{RESULTS}

Modeling results were used to guide experiments for process optimization using the following strategy: (i) generate experimental and model results under specific test conditions; (ii) confirm model accuracy by comparing with experimental results; (iii) explore the predicted effects of composition and processing variables on final target properties by running the model under various new conditions; (iv) identify optimum initial fluid compositions and processing conditions, and ( $v$ ) carry out experiments near predicted optimum conditions.

To test the reliability of the model, experimental shell production and simulated runs were performed under the same conditions. The inputs to the model included standard reference values of pure component densities and pair saturation weight fractions. Fujita-Doolittle parameters $(A$ and $B$ ) were taken from literature values 7 on other polymer systems; these parameters were assumed to hold for the organic solvents used in this work. $D_{0}$ values were selected to yield $1 \times 10^{-5}$ (order of magnitude of liquid self-diffusivity) at infinite polymer dilution. Therefore, the diffusivities of the components are assumed to have the same dependence on polymer concentration.

Experimental shell behavior was measured by producing shells in a special viewing column. Individual shells were suspended by a metered flow of external fluid, video taped during the solidification process, and then analyzed with frame-grabbing software. The dimensional evolution of a test system with wall fluid consisting of polystyrene dissolved in methylethylketone/1,2-dichloroethane/benzene at $40^{\circ} \mathrm{C}$ is shown in Figure 2. The prediction of wall thinning compares well with experimental results. 


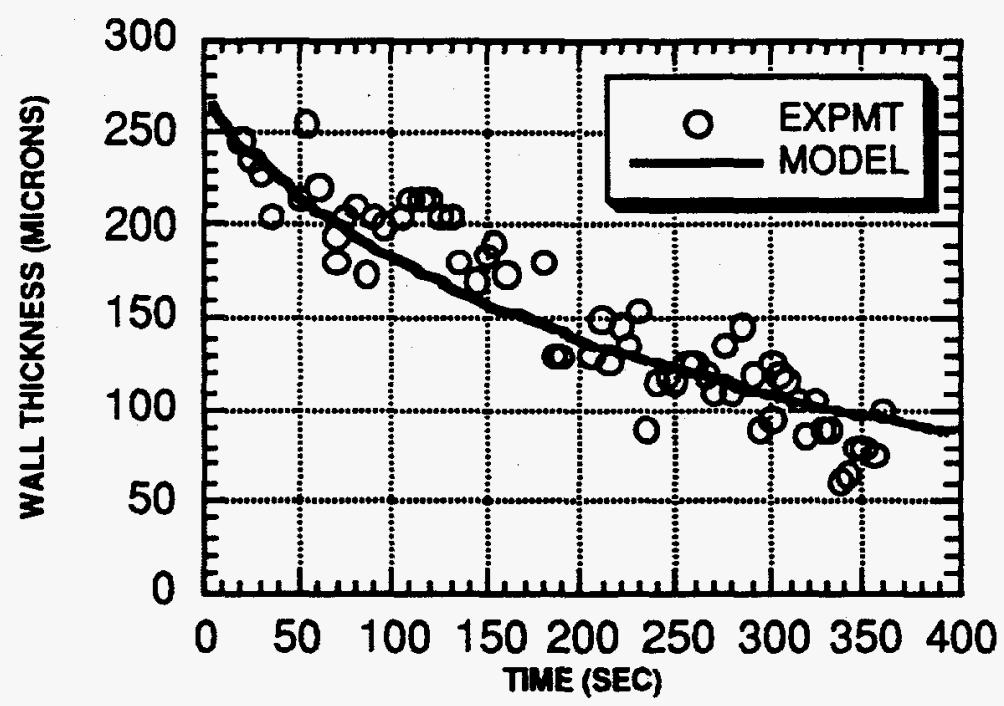

Figure 2. Comparison of experimental and simulated wall thinning of a shell produced with 0.05 polystyrene $(90,000 \mathrm{MW}) / 0.30$ methyl-ethyl ketone/0.55 1,2 dichloroethane $/ 0.10$ benzene (weight fraction) at $40^{\circ} \mathrm{C}$.

Predictions of the wall density trajectory showed relatively large $(>0.05)$ core/wall density differences during solidification (see Figure 3 ). This suggested that there may be severe core/wall nonconcentricity (core offset); in the past, only the method of agitation was presumed to influence concentricity. Further analysis of the video images confirmed that the core position does vary significantly prior to solidification. The experimental window of core/wall density-matching was accurately predicted by the model, as shown in Figure 3.

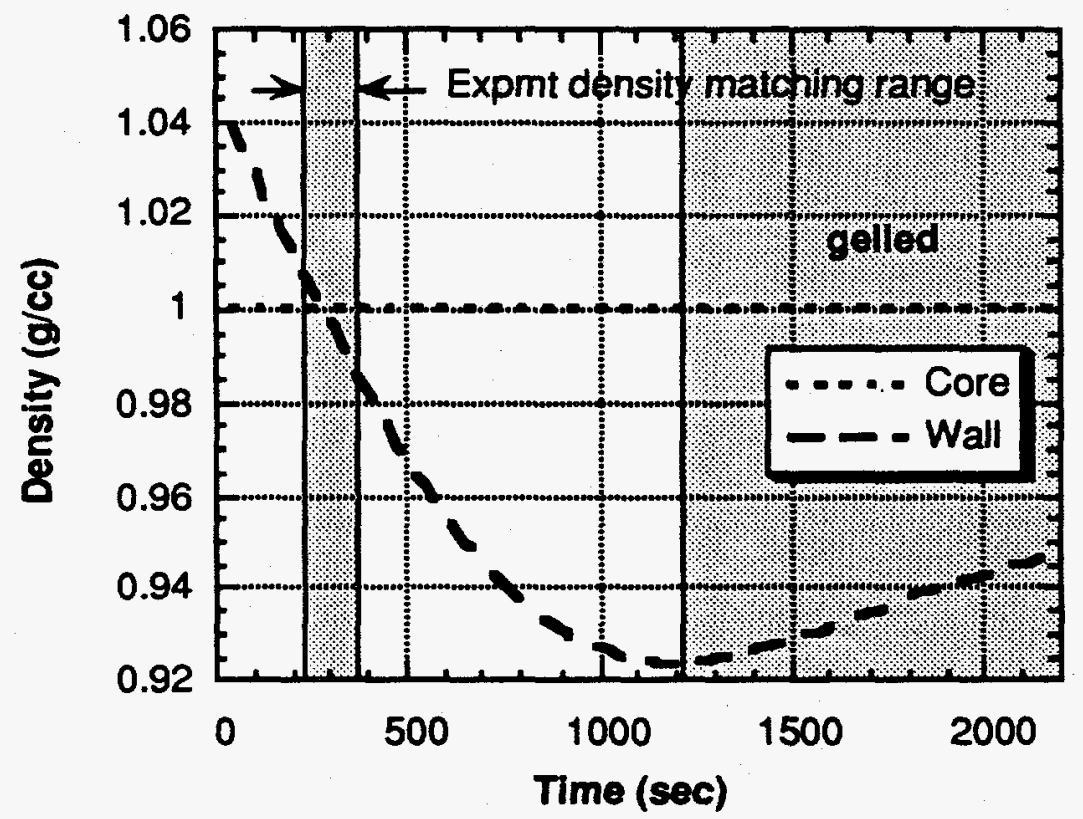

Figure 3. Comparison of experimental and simulated core/wall density-matching for shells produced with 0.10 polystyrene $(90,000 \mathrm{MW}) / 0.531,2$-dichloroethane/ 0.37 toluene (weight fraction) at $65^{\circ} \mathrm{C}$; the wall gels at $=40 \%$ polymer.

If shells solidify when the core/wall density difference is significant, one would expect poor concentricity to result. Therefore, we employed the model to efficiently explore many initial wall compositions and processing temperatures which would result in core/wall density-matching just 
prior to shell solidification ( $\approx 40 \%$ polymer by viscometry). Conditions which met this objective were identified and then implemented experimentally. Shell production runs at conditions close to these yielded significantly higher $(99 \%)$ concentricity than achieved prior to the modeling effort $(65 \%)$, bringing shell concentricity up to target standards.

To address the vacuole problem, the evolution of trace amounts of water entering into the shell wall was tracked in the simulations. Figure 4 shows the water concentration trajectory and its proximity to phase envelope estimations from the modified UNIFAP calculations. Initially, trace amounts of water diffuses into the organic-rich wall. As the wall becomes polymer-rich, water is rejected back out into the external fluid.

If the trajectory does actually cross into the 2-phase region, microphase separation may occur. Considering the simplifying assumptions within the model, these results suggest that microphase separation is a reasonable explanation for vacuole formation. In the future, the full multicomponent diffusion formalism described above will be implemented to produce more reliable predictions of the water dynamics and thermodynamics. The model can then be used to explore processing and compositional conditions which suppress vacuole formation.

\section{ACKNOWLEDGMENTS}

This work was performed under the auspices of the U.S. Department of Energy by Soane Technologies, Inc., under Contract DE-AC03-91SF18601, and by Lawrence Livermore National Laboratory under Contract W-7405-ENG-48.

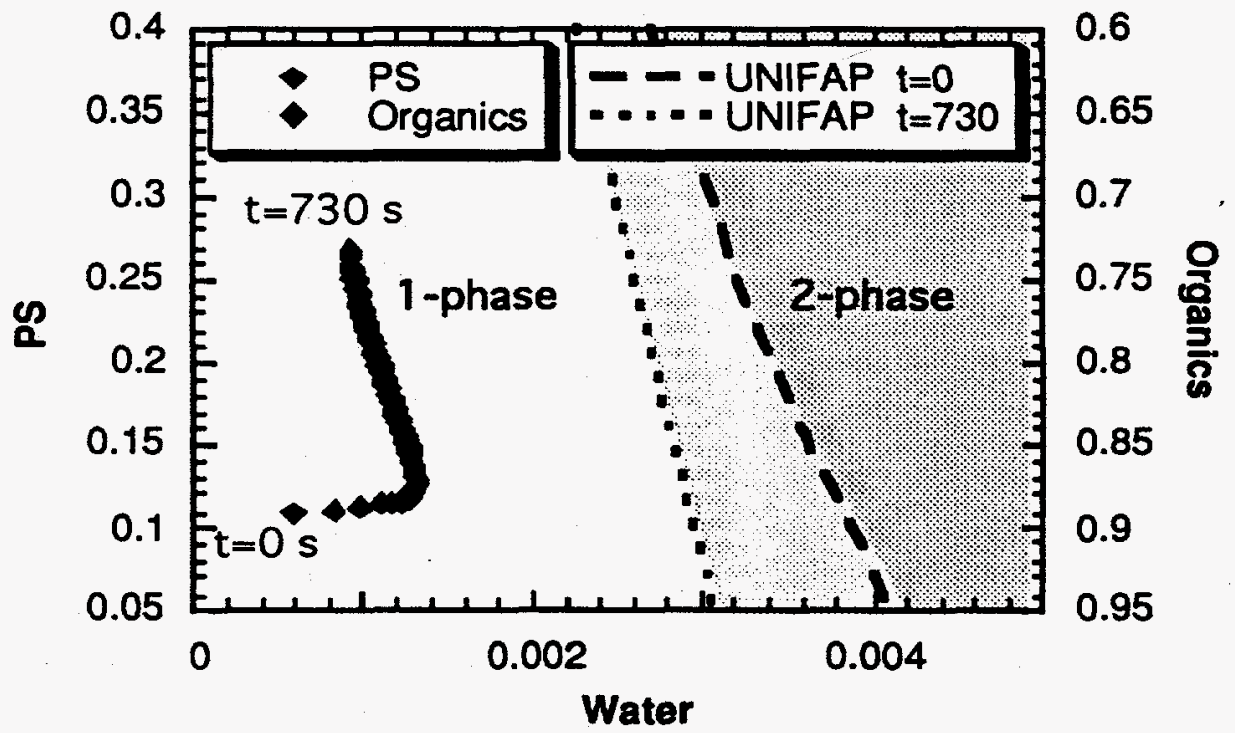

Figure 4. Simulated dynamic water weight fraction within a wall initially consisting of 0.10 polystyrene $(90,000 \mathrm{MW}) / 0.53$ 1,2-dichloroethane $/ 0.37$ toluene (weight fraction) at $77^{\circ} \mathrm{C}$, along with UNIFAP predictions for polystyrene/1,2-dichloroethane/toluene/ water, the two-phase region is to the right of each UNIFAP curve.

\section{REFERENCES}


1. R. Cook, "Production of Hollow Microspheres for Inertial Confinement Fusion Experiments," this volume.

2. K.E. Gubbins, in Statistical Mechanics, (A Specialist Periodical Report, The Chemical Society 1, London, 1973) p. 194.

3. A.Z. Akcasu, in Dynamic Light Scattering, edited by W. Brown, (Clarendon Press, Oxford, 1993) p. 1.

4. J.M. Prausnitz, T.F. Anderson, E.A. Grens, in Computer Calculations for Multicomponent Vapor-Liquid and Liquid-Liquid Equilibria. (Prentice-Hall, Inc., Englewood Cliffs, New Jersey, 1980).

5. R.B. Bird, W.E. Stewart, and E.N. Lightfoot in Transport Phenomena, (John \& Sons, New York, 1960), p. 647.

6. L. Lapidus, G. Pinder, in Numerical Solution of Partial Differential Equations in Science and Engineering, (John Wiley, New York, 1982), p. 50.

7. B.M. Louie, G.M. Carratt, D.S. Soong, J. Appl. Poly. Sci. 30, 3985 (1985). 